\title{
New Legume Hosts of Phakopsora pachyrhizi Based on Greenhouse Evaluations
}

\author{
T. L. Slaminko, Department of Crop Sciences, University of Illinois, Urbana 61801; M. R. Miles, United States \\ Department of Agriculture-Agricultural Research Service (USDA-ARS), Urbana, IL 61801; R. D. Frederick and \\ M. R. Bonde, USDA-ARS Foreign Disease-Weed Science Research Unit, Ft. Detrick, MD 21702; and G. L. Hartman, \\ USDA-ARS and Department of Crop Sciences, University of Illinois, Urbana 61801
}

\begin{abstract}
Slaminko, T. L., Miles, M. R., Frederick, R. D., Bonde, M. R., and Hartman, G. L. 2008. New legume hosts of Phakopsora pachyrhizi based on greenhouse evaluations. Plant Dis. 92:767-771.

Phakopsora pachyrhizi, the causal organism of soybean rust, was first found in the continental United States in 2004 and has been found on soybean, kudzu, Florida beggarweed, and three Phaseolus species in the field. The pathogen has been reported to occur on more than 90 legume species worldwide and it is likely to infect native and introduced legume species in the United States. The objective of this study was to determine if 176 species representing 57 genera of legumes, the majority of which are either native or naturalized to soybean-growing areas of the United States, could be hosts of P. pachyrhizi. Between one and three accessions of each species, a total of 264 accessions, were inoculated with a mixture of four isolates of $P$. pachyrhizi. Severity and sporulation were rated on a 1-to-5 scale at 14 and 28 days after inoculation. P. pachyrhizi was confirmed by the presence of sporulating uredinia and/or immunological assay on 65 new species in 25 genera; 12 of these genera have not been reported previously as hosts. Many of the newly identified hosts grow in the southern United States, and like kudzu, could serve as overwintering hosts for P. pachyrhizi.
\end{abstract}

Additional keywords: host range, Papilionoideae

Soybean rust is caused by the obligate fungus Phakopsora pachyrhizi Syd. \& P. Syd. and was first reported in Asia (10). It is a serious foliar disease on soybean in Africa, Asia, Australia, and South America (15), but its impact on soybean production in North America has been limited since its arrival in 2004 (22) to the southern United States (24).

There are 93 hosts of $P$. pachyrhizi reported from inoculated and noninoculated plants $(12,17,21,23)$. All hosts belong to the legume family Fabaceae, which is one of the largest families of flowering plants

Corresponding author: G. L. Hartman

E-mail: ghartman@uiuc.edu

Trade and manufacturers' names are necessary to report factually on available data; however, the USDA neither guarantees nor warrants the standard of the product, and the use of the name by USDA implies no approval of the product to the exclusion of others that may also be suitable.

*The $e$-Xtra logo stands for "electronic extra" and indicates that a supplementary figure and table not included in the print edition are available online.

Accepted for publication 8 January 2008

doi:10.1094/PDIS-92-5-0767

This article is in the public domain and not copyrightable. It may be freely reprinted with customary crediting of the source. The American Phytopathological Society, 2008. containing over 650 genera and 18,000 species (18). The host range of $P$. pachyrhizi is restricted to the subfamily Papilionoideae, a monophyletic clade within Fabaceae (26). The subfamily contains an estimated 476 genera and 13,860 species (5). $P$. pachyrhizi is known to infect 42 genera of papilionoid legumes, but the limits of its host range are not known. Legume systematics might provide useful insight on the potential host range of $P$. pachyrhizi and its relationship with host plants.

In the United States, six species have been reported as hosts of $P$. pachyrhizi: soybean (22); kudzu, Pueraria lobata (Willd.) Ohwi (8); Florida beggarweed, Desmodium tortuosum (Sw) DC. (23); dry bean, Phaseolus vulgaris L.; lima bean, $P$. lunatus L.; and scarlet runner bean, $P$. coccineus L. (12).

With the appearance of soybean rust in the continental United States in 2004 (22), there are many new potential legume hosts of $P$. pachyrhizi. Many legume species in the United States were either not previously tested or were not in the same geographic region as the fungus. These leguminous species may aid in the overwintering of the fungus and provide a source of inoculum at the beginning of the soybean-growing season. The objective of this study is to identify leguminous hosts that are either native or naturalized to the southern United States or other major soybean-growing areas.

\section{MATERIALS AND METHODS}

Species selection. All species tested belong to the legume subfamily Papilionoideae and all occur in the southern United States or in major soybean-producing states (25) with the exception of previously reported hosts and four unscreened species in Teramnus, the genus most closely related to Glycine (11). Seed was ordered from several repositories in the USDAARS National Plant Germplasm System (Table 1).

Experimental procedures. Between 2 and 20 seeds per entry per replication were planted into each $4-\mathrm{cm}^{2}$ cell in $6 \times 12$ flats containing soilless medium (Sunshine Mix, LC1; Sun Gro Horticulture Inc., Bellevue, WA) in a rust-free greenhouse. Seed number varied because of seed size, availability, germination, and plant size. Seeds of some species were scarified or pregerminated on water agar to improve the likelihood of survival. The experimental design was a randomized complete block with three replications. Two soybean cultivars, GC00138-29 and UG-5, were included in each replication as susceptible checks. The experimental unit was a cell containing 1 to 20 plants of a single entry, which represented a unique plant introduction (PI).

Inoculation. Four-week-old plants were inoculated with a mixture of four isolates of $P$. pachyrhizi at the USDA-ARS Foreign Disease-Weed Science Research Unit (FDWSRU) Biosafety Level 3 Plant Pathogen Containment Facility at Ft. Detrick, MD (13). The inoculum consisted of an equal mixture of isolates from Brazil (BZ 01-1), Paraguay (PG 01-2), Thailand (TH 01-1), and Zimbabwe (ZM 01-1) (14). Spores were removed from liquid nitrogen storage, heat shocked at $40^{\circ} \mathrm{C}$ for $5 \mathrm{~min}$, and allowed to rehydrate by incubating over water in an enclosed petri plate for 12 h (14). Distilled water with $0.01 \%$ (v/v) Tween 20 (Sigma, St. Louis, MO) was added to the dry spores and the mixture was stirred with a glass rod. The spore suspension was then filtered through a 53$\mu \mathrm{m}$ nylon screen into a beaker to remove debris and clumps of spores, and the spore concentration was determined with a hemacytometer. Distilled water was added to adjust the final spore concentration to 25,000 spores per $\mathrm{ml}$. Each flat was inoculated with $25 \mathrm{ml}$ of the spore suspension with an atomizer at $138 \mathrm{kPa}$. Flats were 
placed in a dew chamber at 20 to $22^{\circ} \mathrm{C}$ for $16 \mathrm{~h}$ and then transferred to a greenhouse at 20 to $25^{\circ} \mathrm{C}$ with a 16 -h photoperiod. Supplemental lighting was provided with 1,000-watt Metalarc high-intensity lamps
(Sylvania, Danvers, MA). The plants were rated 14 days after inoculation (DAI) and reinoculated with the same procedure as described above. After incubation in the dew chamber, the plants were placed in a mist chamber in the greenhouse at 20 to $25^{\circ} \mathrm{C}$ with natural light. Mist was provided for $1 \mathrm{~min}$ at $20 \mathrm{~min}$ intervals for the duration of the experiment. The plants were rated as before at $14 \mathrm{DAI}$.

Table 1. List of legume hosts that were susceptible when inoculated with Phakopsora pachyrhizi in the greenhouse and the lesion type and severity ratings for each species

\begin{tabular}{|c|c|c|c|c|c|c|c|c|c|}
\hline \multirow[b]{2}{*}{ Scientific name } & \multirow[b]{2}{*}{ Accession $^{\text {b }}$} & \multirow[b]{2}{*}{$\begin{array}{c}\text { Seed } \\
\text { source }\end{array}$} & \multirow[b]{2}{*}{$\begin{array}{c}\text { New } \\
\text { genus }^{d}\end{array}$} & \multirow[b]{2}{*}{$\begin{array}{c}\text { New } \\
\text { species }^{d}\end{array}$} & \multirow[b]{2}{*}{$\begin{array}{l}\text { Lesion } \\
\text { type }^{\mathrm{e}}\end{array}$} & \multirow[b]{2}{*}{ No. ${ }^{f}$} & \multicolumn{3}{|c|}{ Severity ${ }^{a}$} \\
\hline & & & & & & & Mean & Range & $\begin{array}{c}\text { Standard } \\
\text { error }\end{array}$ \\
\hline Alysicarpus rugosus & PI 286530 & S9 & & $\mathrm{x}$ & $\mathrm{RB}$ & 9 & 1.9 & $1-4$ & 0.39 \\
\hline Astragalus canadensis & PI 232539 & W6 & $\mathrm{x}$ & & $\mathrm{RB}$ & 8 & 1.8 & $1-3$ & 0.25 \\
\hline A. cicer & $\begin{array}{l}\text { PI } 362119^{\mathrm{a}}, \text { PI } 452451^{\mathrm{a}}, \\
\text { PI } 576968^{\mathrm{a}}\end{array}$ & W6 & & & $\mathrm{BL}, \mathrm{RB}$ & 16 & 3.2 & $2-4$ & 0.16 \\
\hline A. crassicarpus & DLEG 900280 & DLEG & & & $\mathrm{RB}$ & 4 & 1.3 & $1-2$ & 0.25 \\
\hline A. glycyphyllos & PI 206882, PI 420665 & W6 & & & RB & 10 & 1.6 & $1-2$ & 0.16 \\
\hline $\begin{array}{l}\text { Baptisia alba var. } \\
\text { macrophylla }\end{array}$ & Ames 27423ª, PI 636376 & NC7 & $\mathrm{x}$ & $\mathrm{x}$ & $\mathrm{RB}$ & 10 & 2.0 & $1-3$ & 0.26 \\
\hline B. australis & Ames 24958, PI 443123 & NC7 & & $\mathrm{x}$ & $\mathrm{BL}, \mathrm{RB}$ & 11 & 1.5 & $1-3$ & 0.21 \\
\hline $\begin{array}{l}\text { B. bracteata } \text { var. } \\
\text { laevicaulis }\end{array}$ & Ames $3095^{\mathrm{a}}$ & NC7 & & $\mathrm{x}$ & $\mathrm{BL}, \mathrm{RB}$ & 6 & 2.5 & $1-3$ & 0.34 \\
\hline Cajanus cajan & NSL 73128, PI 520598 & NPGS & & & $\mathrm{BL}, \mathrm{RB}$ & 9 & 3.6 & $2-5$ & 0.38 \\
\hline Calopogonium caeruleum & PI $362125^{\mathrm{a}}$ & S9 & & & $\mathrm{RB}, \mathrm{TAN}$ & 5 & 4.0 & $3-5$ & 0.45 \\
\hline $\begin{array}{l}\text { Calopogonium } \\
\text { mucunoides }\end{array}$ & $\begin{array}{l}\text { PI } 204364^{\mathrm{a}}, \text { PI } 279595^{\mathrm{a}}, \\
\text { PI } 286288^{\mathrm{a}}, \text { PI } 322302^{\mathrm{a}}\end{array}$ & S9 & & $\mathrm{x}$ & $\mathrm{RB}, \mathrm{TAN}$ & 24 & 3.1 & $2-5$ & 0.15 \\
\hline Caragana arborescens & $\begin{array}{l}\text { PI } 310390^{\mathrm{a}} \text {, PI } 369217^{\mathrm{a}}, \\
\text { PI } 371524^{\mathrm{a}}, \text { PI } 633648^{\mathrm{a}} \text {, } \\
\text { PI } 636378^{\mathrm{a}}\end{array}$ & NC7 & $\mathrm{x}$ & $\mathrm{x}$ & $\mathrm{RB}$ & 27 & 1.9 & $1-3$ & 0.14 \\
\hline Centrosema virginianum & PI $322350^{a}$, PI $386281^{\mathrm{a}}$ & S9 & & $\mathrm{x}$ & $\mathrm{BL}, \mathrm{RB}$ & 8 & 1.9 & $1-4$ & 0.35 \\
\hline $\begin{array}{l}\text { Cologania angustifolia } \\
\text { var. angustifolia }\end{array}$ & $\begin{array}{l}\text { DLEG 900669a }, \text { DLEG } \\
990127^{\mathrm{a}}\end{array}$ & DLEG & $\mathrm{x}$ & $\mathrm{x}$ & RB, RED & 12 & 3.7 & $2-5$ & 0.26 \\
\hline $\begin{array}{l}\text { Cologania angustifolia } \\
\text { var. stricta }\end{array}$ & $\begin{array}{l}\text { DLEG 890367Da, } \\
\text { DLEG 990130a }\end{array}$ & DLEG & & $\mathrm{x}$ & $\mathrm{RB}, \mathrm{TAN}$ & 11 & 3.1 & $2-4$ & 0.21 \\
\hline Cologania lemmonii & DLEG $880053 \mathrm{D}^{\mathrm{a}}$ & DLEG & & $\mathrm{x}$ & RB & 1 & 4.0 & 4 & \\
\hline Crotalaria incana & PI 263427a , PI 336996a & S9 & & $\mathrm{x}$ & RB, TAN & 14 & 1.8 & $1-3$ & 0.19 \\
\hline C. lanceolata & PI $322408^{a}$ & S9 & & $\mathrm{x}$ & TAN, NF & 5 & 1.6 & $1-3$ & 0.40 \\
\hline C. ochroleuca & PI 274767, PI 543869 $9^{\mathrm{a}}$ & S9 & & $\mathrm{x}$ & $\mathrm{RB}, \mathrm{NF}$ & 14 & 1.7 & $1-3$ & 0.19 \\
\hline C. pallida & PI 189272 & S9 & & $\mathrm{x}$ & $\mathrm{RB}$ & 6 & 1.2 & $1-2$ & 0.17 \\
\hline C. sagittalis & DLEG $900645^{\mathrm{a}}$ & DLEG & & $\mathrm{x}$ & $\mathrm{RB}$ & 6 & 1.5 & $1-2$ & 0.22 \\
\hline C. spectabilis & PI 240413, PI 407529 & S9 & & & $\mathrm{BL}, \mathrm{RB}, \mathrm{NF}$ & 12 & 1.7 & $1-2$ & 0.14 \\
\hline C. verrucosa & PI 209316a & S9 & & $\mathrm{x}$ & $\mathrm{BL}, \mathrm{RB}$ & 6 & 1.8 & $1-3$ & 0.31 \\
\hline $\begin{array}{l}\text { C. virgulata ssp. } \\
\text { grantiana }\end{array}$ & PI 68849 & S9 & & $\mathrm{x}$ & $\mathrm{BL}, \mathrm{RB}$ & 2 & 3.0 & 3 & 0.00 \\
\hline Desmodium canadense & PI 214108 & S9 & & $\mathrm{x}$ & $\mathrm{RB}$ & 3 & 1.7 & $1-3$ & 0.67 \\
\hline D. cuspidatum & PI $214105^{\mathrm{a}}$ & S9 & & $\mathrm{x}$ & $\mathrm{RB}$ & 4 & 2.0 & $1-3$ & 0.41 \\
\hline D. obtusum & PI $316210^{\mathrm{a}}$ & S9 & & $\mathrm{x}$ & $\mathrm{RB}$ & 6 & 3.0 & $2-4$ & 0.45 \\
\hline D. perplexum & PI $322465^{\mathrm{a}}$ & S9 & & $\mathrm{x}$ & $\mathrm{RB}$ & 6 & 2.7 & $2-4$ & 0.33 \\
\hline Genista tinctoria & PI 325343, PI 502384 & W6 & $\mathrm{x}$ & $\mathrm{x}$ & $\mathrm{RB}$ & 16 & 1.8 & $1-4$ & 0.26 \\
\hline Glycine max & GC00138-29a, UG-5 ${ }^{\mathrm{a}}$ & UIUC & & & RB, TAN & 12 & 3.3 & $2-5$ & 0.25 \\
\hline Glycyrrhiza lepidota & $\begin{array}{l}\text { PI } 215212^{\mathrm{a}}, \text { PI } 215213, \\
\text { PI } 215215^{\mathrm{a}}\end{array}$ & W6 & $\mathrm{x}$ & $\mathrm{x}$ & $\mathrm{BL}, \mathrm{RB}$ & 18 & 2.3 & $1-3$ & 0.20 \\
\hline Indigofera miniata & PI 477963 & S9 & $\mathrm{x}$ & $\mathrm{x}$ & $\mathrm{RB}$ & 12 & 1.3 & $1-3$ & 0.19 \\
\hline I. spicata & PI $257752^{\mathrm{a}}$ & S9 & & $\mathrm{x}$ & $\mathrm{BL}, \mathrm{RB}$ & 10 & 2.0 & $1-4$ & 0.33 \\
\hline I. suffruticosa & PI 206323, PI 331110 & S9 & & $\mathrm{x}$ & $\mathrm{RB}$ & 10 & 2.1 & $1-3$ & 0.28 \\
\hline I. tinctoria & PI 198005, PI 300006 & S9 & & $\mathrm{x}$ & $\mathrm{RB}$ & 12 & 1.5 & $1-3$ & 0.23 \\
\hline Kummerowia stipulacea & $\begin{array}{l}\text { PI } 186584^{\mathrm{a}} \text {, PI } 295943^{\mathrm{a}}, \\
\text { PI } 419958\end{array}$ & S9 & & & $\mathrm{RB}$ & 18 & 2.6 & $1-5$ & 0.22 \\
\hline K. striata & PI 419960 & S9 & & & $\mathrm{BL}, \mathrm{RB}$ & 14 & 3.4 & $1-5$ & 0.33 \\
\hline $\begin{array}{l}\text { Lablab purpureus ssp. } \\
\text { uncinatus }^{\mathrm{g}}\end{array}$ & PI 532672 & S9 & & & $\mathrm{BL}, \mathrm{RB}$ & 5 & 2.6 & $2-3$ & 0.24 \\
\hline Lathyrus aphaca & $\begin{array}{l}\text { PI } 227511^{\mathrm{a}}, \text { PI } 283485^{\mathrm{a}} \text {, } \\
\text { PI } 358856^{\mathrm{a}}\end{array}$ & W6 & $\mathrm{x}$ & $\mathrm{x}$ & $\mathrm{BL}, \mathrm{RB}$ & 13 & 2.3 & $1-4$ & 0.33 \\
\hline \multirow[t]{2}{*}{ Lathyrus sylvestris } & PI 383275 & W6 & & $\mathrm{x}$ & $\mathrm{RB}$ & 14 & 1.1 & $1-2$ & 0.10 \\
\hline & & & & & & & \multicolumn{3}{|c|}{ (continued on next page } \\
\hline
\end{tabular}

a Severity was based on a 1 -to- 5 scale, in which $1=$ no reaction and $5=$ a severe response.

$\mathrm{b}$ Accessions observed with uredinia and urediniospores.

${ }^{c}$ DLEG $=$ Desert Legume Program, Tucson, AZ; NC7 = North Central Regional Plant Introduction Station, Ames, IA; NPGS = National Center for Genecic Resources Preservation; S9 = Plant Genetic Resources Conservation Unit, Griffin, GA; W6 = Western Regional Plant Introduction Station, Pullman, WA; and UIUC = University of Illinois, Urbana, IL.

d An " $\mathrm{x}$ " indicates that this is the first report of the genus and/or species as compared with Ono et al. (17).

${ }^{\mathrm{e}}$ Lesion types observed on one or more entries and often observed in combination on a single entry. $\mathrm{BL}=$ black; $\mathrm{BR}=$ brown; $\mathrm{NF}=$ light-colored necrotic flecks; $\mathrm{RB}=$ reddish brown; $\mathrm{RED}=$ red; and TAN = tan.

${ }^{\mathrm{f}}$ Number of entries evaluated.

$\mathrm{g}$ This is the first report of the subspecies as compared with a previous report (17). 
Evaluation. Lesion type, disease severity, and sporulation were assessed for each entry. Lesions were rated as either tan (TAN), reddish brown (RB), or a mixture of both (mixed) $(1,2)$. Lesion types that differed from the standard TAN or RB were noted. Disease severity was based on a 1-to-5 scale, in which $1=$ no visible lesions, 2 = few scattered lesions, 3 = moderate number of lesions on at least part of the leaf, $4=$ abundant number of lesions on at least part of the leaf, and $5=$ prolific lesion development over most of the leaf (14). Sporulation within lesions was evaluated as none, some, or abundant. Abundant

Table 1. (continued from preceding page)

\begin{tabular}{|c|c|c|c|c|c|c|c|c|c|}
\hline \multirow[b]{2}{*}{ Scientific name } & \multirow[b]{2}{*}{ Accession $^{a}$} & \multirow[b]{2}{*}{$\begin{array}{c}\text { Seed } \\
\text { source }^{b}\end{array}$} & \multirow[b]{2}{*}{$\begin{array}{c}\text { New } \\
\text { genus }^{c}\end{array}$} & \multirow[b]{2}{*}{$\begin{array}{c}\text { New } \\
\text { species }^{c}\end{array}$} & \multirow[b]{2}{*}{$\begin{array}{l}\text { Lesion } \\
\text { type }^{d}\end{array}$} & \multirow[b]{2}{*}{ No. ${ }^{\mathrm{e}}$} & \multicolumn{3}{|c|}{ Severity $^{f}$} \\
\hline & & & & & & & Mean & Range & $\begin{array}{l}\text { Standard } \\
\text { error }\end{array}$ \\
\hline Lespedeza bicolor & PI 561142 & S9 & & & RB & 4 & 2.0 & $1-3$ & 0.41 \\
\hline L. capitata & $\begin{array}{l}\text { PI } 215225^{\mathrm{a}}, \text { PI } 287114^{\mathrm{a}}, \\
\text { PI } 340795\end{array}$ & S9 & & $\mathrm{x}$ & $\mathrm{RB}$ & 18 & 2.8 & $1-5$ & 0.24 \\
\hline L. cuneata & $\begin{array}{l}\text { PI } 286451^{\mathrm{a}}, \text { PI } 419955^{\mathrm{a}}, \\
\text { PI } 597489^{\mathrm{a}}\end{array}$ & S9 & & $\mathrm{x}$ & $\begin{array}{l}\text { BL, RB, } \\
\text { RED }\end{array}$ & 18 & 2.3 & $1-3$ & 0.16 \\
\hline L. cyrtobotrya & PI $295323^{a}$, PI $360903^{a}$ & S9 & & $\mathrm{X}$ & $\begin{array}{l}\text { BL, RB, } \\
\text { RED }\end{array}$ & 16 & 2.3 & $1-3$ & 0.17 \\
\hline L. thunbergii & PI $360908^{a}$ & S9 & & $\mathrm{x}$ & $\mathrm{BL}, \mathrm{RB}$ & 10 & 1.7 & $1-2$ & 0.15 \\
\hline L. virgata & PI 218004a , PI 349428 & S9 & & $\mathrm{x}$ & $\mathrm{RB}$ & 8 & 1.8 & $1-3$ & 0.25 \\
\hline Lotus corniculatus & PI 568200 & W6 & & & $\mathrm{RB}$ & 18 & 1.2 & $1-2$ & 0.09 \\
\hline $\begin{array}{l}\text { Lotus glaber } \\
\quad(\text { synonym }=\text { L. tenuis })\end{array}$ & PI 246737 & W6 & & $\mathrm{x}$ & $\mathrm{RB}$ & 12 & 1.2 & $1-2$ & 0.11 \\
\hline Lotus pedunculatus & PI $631960^{a}$ & W6 & & $\mathrm{x}$ & $\mathrm{RB}$ & 6 & 1.3 & $1-3$ & 0.33 \\
\hline Lotus unifoliolatus & PI 215235, PI 631744 & W6 & & & RB, RED & 10 & 1.7 & $1-4$ & 0.30 \\
\hline Lupinus albus & $\begin{array}{l}\text { PI } 381322, \text { PI } 481554^{a} \text {, } \\
\text { PI } 502651\end{array}$ & W6 & & & $\mathrm{RB}$ & 13 & 1.9 & $1-3$ & 0.18 \\
\hline Lupinus angustifolius & $\begin{array}{l}\text { PI } 168527^{\mathrm{a}}, \text { PI } 383249^{\mathrm{a}} \text {, } \\
\text { PI } 384551, \text { PI } 615400\end{array}$ & W6 & & & $\mathrm{RB}$ & 23 & 1.9 & $1-2$ & 0.06 \\
\hline Lupinus luteus & $\begin{array}{l}\text { PI } 168544^{\mathrm{a}}, \text { PI } 224493, \\
\text { PI } 505850^{\mathrm{a}}, \text { PI } 533713^{\mathrm{a}}\end{array}$ & W6 & & & $\mathrm{RB}$ & 22 & 1.7 & $1-2$ & 0.10 \\
\hline Lupinus perennis & DLEG $920280^{\mathrm{a}}$ & DLEG & & $\mathrm{x}$ & $\mathrm{RB}, \mathrm{TAN}$ & 2 & 2.0 & 2 & 0.00 \\
\hline Lupinus texensis & DLEG 910497ª & DLEG & & $\mathrm{x}$ & $\mathrm{RB}$ & 4 & 2.8 & $2-4$ & 0.48 \\
\hline $\begin{array}{l}\text { Macroptilium } \\
\text { atropurpureum }\end{array}$ & PI $311515^{a}$, PI $543380^{a}$ & S9 & & & $\mathrm{RB}$ & 8 & 3.4 & $2-4$ & 0.32 \\
\hline Macroptilium lathyroides & $\begin{array}{l}\text { PI } 276183^{\mathrm{a}}, \text { PI } 292360^{\mathrm{a}}, \\
\text { PI } 330353^{\mathrm{a}}\end{array}$ & S9 & & & $\begin{array}{l}\text { RB, RED, } \\
\text { TAN }\end{array}$ & 10 & 3.2 & $2-4$ & 0.20 \\
\hline Medicago laciniata & PI $516674^{\mathrm{a}}$ & W6 & & $\mathrm{x}$ & $\mathrm{RB}$ & 15 & 1.2 & $1-3$ & 0.14 \\
\hline M. lupulina & PI 631966a, W6 19435 & W6 & & $\mathrm{x}$ & $\mathrm{RB}$ & 16 & 1.2 & $1-2$ & 0.10 \\
\hline M. minima & $\begin{array}{l}\text { PI } 498935, \text { PI } 499137, \\
\text { PI } 537253\end{array}$ & W6 & & $\mathrm{x}$ & $\mathrm{RB}$ & 15 & 1.3 & $1-2$ & 0.13 \\
\hline M. orbicularis & $\begin{array}{r}\text { PI } 249918^{a}, \text { PI } 287236, \\
\text { PI } 535517^{\mathrm{a}}, \text { PI } 535518\end{array}$ & W6 & & $\mathrm{x}$ & $\mathrm{BL}, \mathrm{RB}$ & 29 & 1.9 & $1-4$ & 0.16 \\
\hline M. polymorpha & $\begin{array}{l}\text { PI 535528, W6 4234, } \\
\text { W6 } 5380\end{array}$ & W6 & & $\mathrm{x}$ & $\mathrm{BL}, \mathrm{RB}$ & 18 & 1.9 & $1-4$ & 0.21 \\
\hline M. sativa $\mathrm{ssp}$. falcata & PI 440539a & W6 & & $\mathrm{x}$ & $\mathrm{RB}$ & 17 & 1.2 & $1-2$ & 0.10 \\
\hline M. sativa $\mathrm{ssp}$. sativa & PI 247790, PI 536536 & W6 & & $\mathrm{x}$ & $\mathrm{BL}$ & 36 & 1.1 & $1-2$ & 0.05 \\
\hline Neonotonia wightii & PI 284804, PI 339895a & S9 & & & $\mathrm{RB}, \mathrm{TAN}$ & 12 & 2.7 & $1-4$ & 0.28 \\
\hline Pseudovigna argentea & PI $365594^{\mathrm{a}}$ & S9 & $\mathrm{x}$ & $\mathrm{x}$ & $\mathrm{RB}, \mathrm{TAN}$ & 3 & 4.0 & $3-5$ & 0.58 \\
\hline Robinia pseudoacacia & DLEG 910265 & DLEG & $\mathrm{x}$ & $\mathrm{x}$ & $\mathrm{RB}$ & 7 & 2.4 & $1-5$ & 0.57 \\
\hline R. viscosa var. hartwegii & PI 560156 & NC7 & & $\mathrm{x}$ & $\mathrm{RB}$ & 5 & 2.4 & $1-3$ & 0.24 \\
\hline Senna sophera & DLEG $900003^{\mathrm{a}}$ & DLEG & & $\mathrm{x}$ & $\mathrm{RB}$ & 6 & 2.3 & $1-3$ & 0.21 \\
\hline Sesbania punicea & DLEG 940172 & DLEG & & $\mathrm{x}$ & $\mathrm{BL}, \mathrm{RB}, \mathrm{NF}$ & 7 & 1.4 & $1-3$ & 0.30 \\
\hline S. virgata & PI 175007 & S9 & & $\mathrm{x}$ & $\mathrm{BL}, \mathrm{RB}$ & 6 & 1.8 & $1-2$ & 0.17 \\
\hline Tephrosia cinerea & PI 296078 & S9 & $\mathrm{x}$ & $\mathrm{x}$ & $\mathrm{RB}$ & 2 & 2.5 & $1-3$ & 0.50 \\
\hline Tephrosia purpurea & $\begin{array}{l}\text { PI } 200238^{\mathrm{a}}, \text { PI } 219855, \\
\text { PI } 270391^{\mathrm{a}}, \text { PI } 318815^{\mathrm{a}}\end{array}$ & S9 & & $\mathrm{x}$ & $\mathrm{RB}$ & 15 & 2.5 & $1-3$ & 0.19 \\
\hline Teramnus labialis & $\begin{array}{l}\text { PI } 200233, \text { PI } 365056^{\mathrm{a}} \text {, } \\
\text { PI } 517204^{\mathrm{a}}\end{array}$ & S9 & $\mathrm{x}$ & $\mathrm{x}$ & $\mathrm{BR}, \mathrm{RB}$ & 21 & 2.4 & $1-4$ & 0.18 \\
\hline Teramnus labialis & CU-383 & UIUC & & & RB, RED & 21 & 2.4 & $1-4$ & 0.18 \\
\hline Teramnus micans & CU-414-2 ${ }^{\mathrm{a}}$ & UIUC & & $\mathrm{x}$ & RB & 4 & 2.3 & $1-3$ & 0.25 \\
\hline Teramnus repens & CU-220 & UIUC & & $\mathrm{x}$ & RED & 2 & 2.0 & $1-3$ & 1.00 \\
\hline Teramnus uncinatus & $\begin{array}{l}\text { PI } 241837, \text { PI } 296583, \\
\text { PI } 316041, \text { PI } 321388^{a}\end{array}$ & S9 & & $\mathrm{x}$ & $\mathrm{BL}, \mathrm{RB}$ & 22 & 2.4 & $1-4$ & 0.14 \\
\hline Trifolium aureum & PI $440721^{\mathrm{a}}$ & S9 & & $\mathrm{x}$ & $\mathrm{RB}$ & 6 & 1.3 & $1-2$ & 0.21 \\
\hline T. сегпиит & PI 196307a & S9 & & $\mathrm{x}$ & $\mathrm{BL}, \mathrm{RB}$ & 6 & 2.2 & $1-3$ & 0.31 \\
\hline T. incarnatum & PI $613044^{\mathrm{a}}$ & S9 & & & RB & 17 & 1.6 & $1-5$ & 0.32 \\
\hline T. lappaceum & PI 254917a, PI 517114 & S9 & & $\mathrm{x}$ & RB & 17 & 2.4 & $1-5$ & 0.32 \\
\hline T. reflexum & PI 291825 & S9 & & $\mathrm{x}$ & $\mathrm{RB}$ & 11 & 1.3 & $1-3$ & 0.19 \\
\hline T. resupinatum & PI 445907, PI 517144 & S9 & & $\mathrm{x}$ & RB & 16 & 1.2 & $1-3$ & 0.14 \\
\hline T. striatum & PI 502625 & S9 & & $\mathrm{x}$ & $\mathrm{RB}$ & 6 & 1.5 & $1-3$ & 0.34 \\
\hline T. tomentosum & PI 422494 & S9 & & $\mathrm{x}$ & $\mathrm{RB}$ & 9 & 1.1 & $1-2$ & 0.11 \\
\hline Vigna adenantha & PI 312898, PI 430216a & S9 & & $\mathrm{x}$ & $\mathrm{RB}, \mathrm{NF}$ & 10 & 2.1 & $1-3$ & 0.28 \\
\hline V. luteola & $\begin{array}{l}\text { PI } 196813^{\mathrm{a}}, \text { PI } 355920^{\mathrm{a}}, \\
\text { PI } 406347^{\mathrm{a}}\end{array}$ & S9 & & & $\begin{array}{l}\mathrm{BR}, \mathrm{RB}, \\
\mathrm{RED}\end{array}$ & 18 & 3.1 & $2-4$ & 0.14 \\
\hline V. unguiculata & $\begin{array}{l}\text { PI } 352832^{a}, \text { PI } 578893^{a}, \\
\text { PI } 612519\end{array}$ & S9 & & & $\begin{array}{l}\text { BL, RB, } \\
\text { RED }\end{array}$ & 16 & 2.9 & $1-5$ & 0.20 \\
\hline
\end{tabular}


sporulation was similar to that of the susceptible soybean cultivars used as checks.

Immunoassay. Leaf samples with lesions were excised from each entry at 28 DAI and stored at $-80^{\circ} \mathrm{C}$. At least one sample from each entry was tested for the presence of $P$. pachyrhizi by enzymelinked immunosorbent assay (ELISA) with the Envirologix QualiPlate Kit for Soybean Rust (Envirologix, Portland, ME) and recommended protocol. Tissue samples were prepared by grinding the leaf section with a plastic pestle attached to a power rotary tool in a microcentrifuge tube containing $500 \mu \mathrm{l}$ of QualiPlate Kit extraction buffer. Several negative controls were utilized in the immunoassay. To control for error in the sensitivity of the test kit, 15 entries without visible lesions were randomized among the positive samples. To control for cross-contamination and error in technique, two each of noninoculated soybean leaf samples and plain extraction buffer controls were randomized within each plate. ELISA plates were visually evaluated for a color change to blue, which indicated a positive reaction.

\section{RESULTS}

One hundred fifty-nine entries representing 89 species in 31 genera were visually confirmed to have soybean rust lesions and/or uredinia on at least one of the two rating dates (Table 1). Sixty-five new host species and one new host subspecies were identified, representing 25 genera; 12 genera have not been previously reported. Of the 65 new host species, 39 species had visible uredinia and urediniospores and the remaining had nonsporulating lesions.

Lesion type. The majority of the entries had RB lesions. Only 11 species had TAN lesions: Calopogonium caeruleum, C. mucunoides, Cologania angustifolia var. stricta, Crotalaria incana, Crotalaria lanceolata, Lupinus perennis, Macroptilium lathyroides, Neonotonia wightii, Pseudovigna argentea, Vigna adenantha, and the soybean inoculated controls. Several lesion types that differed from RB or TAN were also observed. These ranged from black, brown, and red to lightly colored necrotic flecks and often occurred in conjunction with the RB lesion type (Table 1).

Sporulation. Sporulation was abundant on all entries that had TAN lesions. Lowto-moderate sporulation was observed on entries with other lesion types with the exception of Caragana arborescens, Cologania lemmonii, Crotalaria incana, Lathyrus aphaca, Lupinus perennis, Medicago lupulina, and Vigna adenantha, which had abundant sporulation on non-TAN lesion types. There was a strong differential sporulation response for several species. For example, on $V$. adenantha, PI 312898 had no sporulation on either rating date while PI 430216 had a high level of sporulation on both dates.
ELISA. Thirty-two species had lesions with no visible sporulation but were ELISA positive for $P$. pachyrhizi. Of the 176 species tested, 87 did not develop lesions for the duration of the trial. Those asymptomatic entries and the negative controls were negative for $P$. pachyrhizi by ELISA.

\section{DISCUSSION}

The host range of $P$. pachyrhizi was summarized in 1992 and consisted of 91 species in Papilionoideae (17). With the introduction of $P$. pachyrhizi to geographical areas outside of the eastern hemisphere, it was likely that the pathogen would encounter and potentially infect additional host species in this same subfamily.

The 65 newly reported host species varied in their response to $P$. pachyrhizi from those with nonsporulating lesions to those with fully sporulating uredinia. The sporulation data suggest some hosts may not be as epidemiologically important as others. Although some plant species had no sporulating uredinia, they are considered hosts because they had become infected by $P$. pachyrhizi and confirmed by ELISA. Sporulation may have been restricted by a number of factors in the trial, including isolate, environmental conditions, duration of experiment, and host genotype and age. On the basis of other studies, the presence of sporulating uredinia may not be a good criterion for identifying a host. For example, accessions in the same host species respond differently when exposed to the same isolate of $P$. pachyrhizi $(3,4,9,16)$. If a host is capable of becoming infected, sporulation may occur under different environmental conditions. Differences in sporulation might serve as an indication of the strength of the host in the epidemiology of the disease. Hosts with lesions that produce urediniospores increase the likelihood for secondary infection or subsequent infection on soybean.

Polymerase chain reaction (PCR) has been used to identify and distinguish between the two very similar soybean rust pathogens, $P$. pachyrhizi and $P$. meibomiae (7). The discovery of $P$. pachyrhizi in South Africa, Argentina, and the United States was confirmed by PCR $(19,20,22)$. When PCR was initially tested with nonsusceptible plants in this trial, it resulted in false positives. The PCR results were also positive for multiple entries showing no symptoms or signs of infection, indicating that the PCR assay was detecting urediniospores on the surface of the leaf. Recently, antibodies have been generated to $P$. pachyrhizi, and an ELISA kit has been developed (Envirologix, Inc.). The ELISA kit was used in this study to confirm infection by $P$. pachyrhizi because it did not react with the urediniospores on the leaf surface of the nonsusceptible host plants.

The exact limits of $P$. pachyrhizi's host range are still uncertain. The host range lies within the monophyletic Papilionoideae subfamily of Fabaceae, but it is unclear if the host range is limited within the subfamily. A more exhaustive screening of papilionoid legumes is needed to determine the host range restrictions. Additionally, while a genus may contain one or more host species, not all the species in that genus may be hosts.

The canavanine-accumulating clade within Papilionoideae is a monophyletic group (26) and includes the majority of known hosts of $P$. pachyrhizi. The exceptions are three genera, Baptisia, Crotalaria, and Lupinus, which belong to the genistoid clade. Legume phylogeny by using Bayesian analysis of the MATK gene showed the genistoid clade as a sister group to the dalbergioid clade, as well as the remaining papilionoids (25). However, parsimony analysis revealed a slightly different phylogenic relationship with the dalbergioid clade branching before the genistoid clade (26). Other host range studies have been used for legume systematics (6), and the host range of $P$. pachyrhizi may be useful in clarifying some relationships in Papilionoideae. If the exception of the genistoid clade is the only other host group outside the canavanineaccumulating clade, it may lend more support to the phylogeny based on parsimony analysis. It can be expected that additional species in the aforementioned genistoid and canavanine-accumulating clades will be susceptible to infection by $P$. pachyr$h i z i$, and closely related groups may also contain hosts.

Of the 65 newly identified species, 62 occur in Alabama, Florida, Louisiana, Mississippi, and Texas, where they may be important for overwintering of the soybean rust pathogen. These include Caragana arborescens, a perennial tree; Crotalaria incana, a perennial forb/ subshrub; and Crotalaria lanceolata, an annual forb. Although none of these species is known to overwinter the pathogen, it is known that kudzu in southern Florida can stay infected year round (8). Knowledge of the host range will help to predict potential overwintering sites and the level of primary inoculum from these sites that might be available to infect soybean fields at the start of the growing season. It will also help define species and prioritize areas to be scouted. Scouting the newly defined host species will result in a greater understanding of their natural infection as well as increased knowledge of their importance in the overwintering of soybean rust in the United States.

\section{ACKNOWLEDGMENTS}

We thank J. Bowers for assistance with the propagation, maintenance, and inoculation of the plants and with the ratings. This research was funded in part by the Illinois Soybean Association, the North Central Soybean Research Program, and the United Soybean Board. 


\section{LITERATURE CITED}

1. Bonde, M. R., Nester, S. E., Austin, C. N., Stone, C. L., Frederick, R. D., Hartman, G. L., and Miles, M. R. 2006. Evaluation of virulence of Phakopsora pachyrhizi and P. meibomiae isolates. Plant Dis. 90:708-716.

2. Bromfield, K. R. 1984. Soybean Rust. American Phytopathological Society, St. Paul, MN.

3. Burdon, J. J. 1987. Phenotypic and genetic patterns of resistance to the pathogen Phakospora pachyrhizi in populations of Glycine canescens. Oecologia 73:257-267.

4. Burdon, J. J., and Marshall, D. R. 1981. Interand intra-specific diversity in the disease-response of Glycine species to the leaf-rust fungus Phakopsora pachyrhizi. J. Ecol. 69:381390.

5. Doyle, J. J., Chappill, J. A., Bailey, C. D., and Kajita, T. 2000. Towards a comprehensive phylogeny of legumes: Evidence from the $r c b \mathrm{~L}$ sequences and non-molecular data. Pages 1-20 in: Advances in Legume Systematics, P. S. Herendeen and A. Bruneau, eds. Royal Botanic Gardens, Kew, Richmond, Surrey, UK.

6. El-Gazzar, A. 1981. Systematic implications of suceptibility to Uromyces rusts in Leguminosae. Pages 979-994 in: Advances in Legume Systematics, R. M. Polhill and P. H. Raven, eds. Royal Botanic Gardens, Kew, Richmond, Surrey, UK.

7. Frederick, R. D., Snyder, C. L., Peterson, G. L., and Bonde, M. R. 2002. Polymerase chain reaction assays for the detection and discrimination of the soybean rust pathogens Phakopsora pachyrhizi and $P$. meibomiae. Phytopathology 92:217-227.

8. Harmon, P. F., Momol, M. T., Marois, J. J., Dankers, H., and Harmon, C. L. 2005. Asian soybean rust caused by Phakopsora pachyrhizi on soybean and kudzu in Florida. Online publication. doi:10.1094/PHP-2005-0613-01RS. Plant Health Progress.

9. Hartman, G. L., Wang, T. C., and Hymowitz, T. 1992. Sources of resistance to soybean rust in perennial Glycine species. Plant Dis. 76:396-399.

10. Hennings, V. P. 1903. A few new Japanese Uredinaceae. Hedwigia 42:(Suppl)107-108.

11. Hymowitz, T. 2004. Speciation and Cytogenetics. Pages 97-136 in: Soybeans: Improvement, Production, and Uses. 3rd ed. Agron. Monogr. 16, H. R. Boerma and J. E. Specht, eds. ASA, CSSA, and SSSA, Madison, WI.

12. Lynch, T. N., Marois, J. J., Wright, D. L., Harmon, P. F., Harmon, C. L., Miles, M. R., and Hartman, G. L. 2006. First report of soybean rust caused by Phakopsora pachyrhizi on Phaseolus spp. in the United States. Plant Dis. 90:970.

13. Melching, J. S., Bromfield, K. R., and Kingsolver, C. H. 1983. The plant pathogen containment facility at Frederick, Maryland. Plant Dis. 67:717-722.

14. Miles, M. R., Frederick, R. D., and Hartman, G. L. 2006. Evaluation of soybean germplasm for resistance to Phakopsora pachyrhizi. Online publication. doi 10.1094/PHP-20060104-01-RS. Plant Health Progress

15. Miles, M. R., Hartman, G. L., and Frederick, R. D. 2003. Soybean rust: Is the U.S. crop at risk? Online publication. APSnet feature, June 2003. Available at http://www.apsnet.org/ online/feature/rust. American Phytopathological Society, St. Paul, MN.

16. Miles, M. R., Pastor-Corrales, M. A., Hartman, G. L., and Frederick, R. D. 2007. Differential response of common bean cultivars to Phakopsora pachyrhizi. Plant Dis. 91:698-704.

17. Ono, Y., Buritica, P., and Hennen, J. F. 1992. Delimitation of Phakopsora, Physopella and Cerotelium and their species on Leguminosae.
Mycol. Res. 96:825-850.

18. Polhill, R. M., Raven, P. H., and Stirton, C. H 1981. Evolution and systematics of the Leguminosae. Pages 1-26 in: Advances in Legumes Systematics, R. M. Polhill and P. H. Raven, eds. Royal Botanic Gardens, Kew, Richmond, Surrey, UK.

19. Pretorius, Z. A., Kloppers, F. J., and Frederick, R. D. 2001. First report of soybean rust in South Africa. Plant Dis. 85:1288.

20. Rossi, R. L. 2003. First report of Phakopsora pachyrhizi, the causal organism of soybean rust in the province of Missiones, Argentina. Plant Dis. 87:102.

21. Rytter, J. L., Dowler, W. M., and Bromfield, K. R. 1984. Additional alternative hosts of Phakopsora pachyrhizi, causal agent of soybean rust. Plant Dis. 68:818-819.

22. Schneider, R. W., Hollier, C. A., Whitman, H. K., Palm, M. E., McKemy, J. M., Hernandez, J. R., Levy, L., and DeVries-Paterson, R. 2005. First report of soybean rust caused by Phakopsora pachyrhizi in the continental United States. Plant Dis. 89:774.

23. Sconyers, L. E., Kemerait, R. C., Brock, J. H. Gitaitis, R. D., Sanders, F. H., Phillips, D. V. and Jost, P. H. 2006. First report of Phakopsora pachyrhizi, the causal agent of Asian Soybean Rust, on Florida beggarweed in the United States. Plant Dis. 90:972.

24. USDA. 2007. Pest Information Platform for Extension and Education. Online publication. Available at www.sbrusa.net.

25. USDA. 2007. The PLANTS Database. Online publication. National Plant Data Center.

26. Wojciechowski, M. F., Lavin, M., and Sanderson, M. J. 2004. A phylogeny of legumes (Leguminosae) based on analysis of the plastid MATK gene resolves many well-supported subclades within the family. Am. J. Bot. 91:1846-1862. 